\title{
EDITORIAL \\ Cervical cancer prevention: The case of developing countries
}

$\mathrm{T}$ his special issue of Salud Publica de Mexico presents an overview of the latest findings on prevention and screening of cervical cancer (CC), as well as new possible approaches to early diagnosis and intervention for controlling this neoplastic disease.

Similar to other developing countries, CC persists in Mexico as a serious public health problem. Three main components to the burden of disease include: the suffering and grief of patients and their families, the side effects of therapy, and its related costs. A positive note in the presence of the high morbidity and mortality caused by CC is that it clearly shows how interdisciplinary cancer research is the best tool to control this disease.

The cause of CC is now known. The presence of human papillomavirus (HPV) in cervical epithelial cells can be unequivocally identified. We now know which viral types pose a risk and the likelihood of neoplasia development. Also, an international effort is ongoing to develop a vaccine that will prevent infection due to HPV oncogenic types.

In Latin America primary prevention is not directed at women only, but also at their sexual partners, who should also take part in health education programs. Only then will control of HPV transmission be possible.

It should be highlighted that inadequate prevention and untimely diagnosis further complicate control of the disease in advanced stages. Evidence observed in various clinical studies shows that patients with cervical cancer should receive chemotherapy together with radiotherapy. This approach results in higher costs and morbidity associated with therapy in advanced disease and compels to conducting cost-benefit studies of this disease.

The different papers included in this issue on CC prevention and screening emphasize the benefits of HPV DNA testing and its high sensitivity. A negative predictive value of $100 \%$ invites to a discussion on how to use it in the population at large, how to finance it, and how these two techniques (cytology and molecular biology) may optimize the periodicity of Pap testing and better identify the population at high risk for CC.

A cautionary message should be noted; vaccine development is still at its early stage. The initial results are encouraging, but also indicate that several years over a decade- should elapse before it is adopted as a primary prevention measure to control CC. Meanwhile, consensus development should help find the best approaches to optimize CC screening programs that incorporate technology developments.

In countries like Mexico, efforts have been made for years to improve the quality and coverage of the Cervical Cancer Screening program. Improving and strengthening this program is indispensable; we have enough leeway to strengthen the current program and to seek the necessary financial resources to incorporate new techniques. Only then will mortality due to CC start to decrease. Mexico has the opportunity to set an example for countries where CC is endemic.

This monograph clearly illustrates how epidemiologic and laboratory research jointly provide concrete, scientifically solid, evidence-based options to control one of the most important public health problems in developing countries. It presents new diagnostic alternatives like visual inspection, as well as an example of the limited access to technology where it is most needed.

What remains to be done? Scientific endeavor has delivered. The next step should be taken by decisionmakers to invest more resources, education, and the necessary means to prevent and control this disease, which devastates thousands of women, along with their families and budgets. If this happens, public health will provide hope long overdue in third world countries.

Alejandro Mohar*

* Instituto Nacional de Cancerología. Avenida San Fernando 22, Tlalpan 14080, México, DF. 